\title{
High Temperature Behaviour of Quantum Mechanical Thermal Functionals
}

\author{
By \\ Ph. Combe*, R. RodrigueZ*, M. Sirugue** and M. Sirugue-Collin***
}

\begin{abstract}
We derive a representation of thermal functionals as expectation with respect to a Markov process in phase space of classical quantities. We prove also that the above quantities have a classical behaviour for high temperature.
\end{abstract}

\section{§1. Introduction}

Path integration proved to be a very useful tool both in statistical mechanics (see e. g. [1], [2], [3], [4], [5]), in non relativistic quantum mechanics (see e.g. [6]) and the references therein) and in constructive field theory (see e.g. [7] and the references therein). Still its field of applications is rapidly growing. It started from the early work of R.P. Feynman ([8], [9]) but became an effective tool when the importance of Wiener (more generally diffusion) processes was recognized in connection with imaginary time Schrödinger equation and Euclidean Field Theory. Only later on (see e.g. [10] for non relativistic quantum mechanics and [11], [12]) for both non relativistic quantum mechanics and relativistic quantum field theory, it was noticed that jump processes could be used in the definition and application of Feynman path integrals i.e. in the real time region.

In the present work we want to show that more general processes containing both a diffusion part and a jump part can be used to study the temperature behaviour of some thermal expectation values of non relativistic quantum mechanical systems. The main tool being a path integral representation of these quantities in terms of purely classical objects. A somewhat different representation has been obtained in [2], while the goals were quite different and more adapted to the zero temperature limit. Our approach is more adapted to the infinite temper-

Communicated by H. Araki, July 26, 1982.

* Université d'Aix-Marseille II and Centre de Physique Théorique, CNRS, Marseille, Cedex 9, France.

** Centre de Physique Théorique, CNRS, Marseille.

*** Université de Provence and Centre de Physique Théorique, CNRS, Marseille. 
ature case.

It is a matter of computation to show that the expectation values of observables in the equilibrium state of a harmonic oscillator at the inverse temperature $\beta=(k T)^{-1}$ satisfies a "heat equation" with respect to a parameter which is a definite combination of the Planck's constant, the inverse temperature $\beta$ and the frequency of the oscillator. This will be made precise in Section 2. This observation allows to derive a path integral representation of these expectation values, actually as an expectation with respect to a Wiener process in phase space of classical functions.

In this paper we show that there exists a similar representation for systems whose hamiltonians are the ones of the harmonic oscillator perturbed by bounded potentials of the trigonometric type.

A very important tool in what follows are the operators introduced by $U$. Fano [13] which allow to give another description of the Weyl quantization. For the sake of completeness we briefly describe these operators and derive one of their most important property (Proposition (2.8)). Moreover we derive for the temperature states of the harmonic oscillator a path integral representation as an expectation value with respect to a Wiener process in the phase space of classical functions (Proposition (2.21)). This allows to describe the various limits of expectation values as $\beta$ goes to zero (or infinity) or $\hbar$ goes to zero (Corollary (2.26) and formula (2.36)).

In Section 3, we study the case where the Hamiltonian of the system is of the form

$$
H=H_{0}+\int_{R^{2 N}} d \mu(a) W_{a}
$$

where $H_{0}$ is the Hamiltonian of a harmonic oscillator, $\mu$ a bounded measure on $\mathbb{R}^{2 N}$ and $W_{a}$ a Weyl operator. We show that if $f$ is a sufficiently regular function on the phase space and $Q(f)$ the corresponding operator according to the Weyl's prescription one has the representation

$$
\begin{gathered}
\operatorname{Tr}\left\{\exp (-\beta H) W_{a}^{*} Q(f) P\right\} \cdot \exp \left\{-\beta|\mu|\left(\boldsymbol{R}^{2 N}\right)\right\} \\
=\mathrm{E}\left[\exp \left\{i \sigma\left(\alpha, \eta_{0}(\beta)\right)\right\} f\left(\xi_{a, 0}(\beta)\right)\right]
\end{gathered}
$$

where $P$ is the parity operator, $b \in \mathbb{R}^{2 N} \rightarrow \sigma(\alpha, b)$ a linear form on the phase space, $\xi_{a,=}$ and $\eta_{\tau}$ are Markov processes with values in $\mathbb{R}^{2 N}$ with mixed diffusion and jump characters.

This is a path integral representation of quantum expectation values as integral over the trajectories of the processes which are functions from $\tau \in[0, \beta]$ to $\mathbb{R}^{2 N}$ with at most singularities of the first kind. This space, namely the space of functions with at most singularities of the first kind has a nice structure of complete separable metric space, and is quite naturally associated with general stochastically continuous processes which are of the type we have considered before. 
The key-tool for the derivation of the previous formula is the observation that the left hand side of (1.2) satisfies with respect to $\beta$ and $a$ an integrodifferential equation which can be identified with the backward Kolmogorov equation of a Markov process which can be easily identified by its stochastic differential equation (Theorem (3.9)). Furthermore the limit for $\beta$ going to zero approaches the same limit as the corresponding quantity for zero potential. This last limit has been studied through in Section 2 .

Finally let us remark that there is a natural extension of the previous techniques to the imaginary time Schrödinger equation of the trigonometric models of relativistic quantum field theory as derived in [14]. We postpone to a forthcoming paper to elaborate on this point.

It is a pleasure for us to acknowledge Prof. G. F. De Angelis for discussions.

\section{§2. Harmonic Oscillator. A Representation of Thermal Expectation Values of Observables}

In this Section we derive a path integral representation of some expectation values of observables in the equilibrium state of a harmonic oscillator at inverse temperature $\beta=(k T)^{-1}$. The integral is the one associated with the standard Wiener process in phase space. It turns out that it gives as a by-product the different limits of these quantities as $\hbar$ goes to zero or the temperature goes to infinity.

Let us consider an $N$ dimensional symmetric harmonic oscillator whose Hamiltonian is given by:

$$
H_{0}=\frac{1}{2} \sum_{i=1}^{N}\left\{\frac{1}{M} P_{\imath}^{2}+M \omega^{2} Q_{\imath}^{2}\right\}
$$

$M>0, \omega>0$. The $Q_{i}$ 's and the $P_{i}$ 's are the usual position and momentum operators. The non symmetric case could be treated as well.

Quantities of interest are built out of the Weyl operators $W_{a}, a \in \mathbb{R}^{2 N}$, which are unitary operators strongly continuous in $a_{j}, j=1,2, \cdots, 2 N$. If $a=(q, p), q$, $p \in \mathbb{R}^{N}$

$$
W_{a}=\exp \left\{i \sum_{i=1}^{N}\left\{q_{i} P_{i}-p_{i} Q_{\imath}\right\}\right\} .
$$

They satisfy the usual canonical commutation relations in the Weyl form:

$$
W_{a} W_{a^{\prime}}=\exp \left\{2 i \pi \sigma\left(a, a^{\prime}\right)\right\} W_{a+a^{\prime}}
$$

where

$$
\sigma\left(a, a^{\prime}\right)=\frac{1}{4 \pi} \sum_{i=1}^{N}\left\{q_{i}^{\prime} p_{i}-q_{i} p_{i}^{\prime}\right\} .
$$

We follow the conventions which can be found for instance in [15].

According to the Weyl prescription, the classical functions $f$ on the phase space can be quantized according to:

$$
Q(f)=(4 \pi)^{-N} \int_{R^{2 N}} d a(\mathscr{I} f)(a) W_{a / 2},
$$


where $\mathscr{I} f$ denotes the Fourier transform of $f$ :

$$
\{\mathscr{I} f\}\left(a^{\prime}\right)=(4 \pi)^{-N} \int_{R^{2 N}} \exp \left\{2 i \pi \sigma\left(a, a^{\prime}\right)\right\} f(a) d a
$$

We do not elaborate on the class of functions which can be quantized according to the above procedure. In what follows it suffices to remark that it works at least for functions which are the Fourier transform of a bounded measure.

Another expression for $Q(f)$ can be given in terms of the operators $W_{a} P$ introduced in [13] (see also [16]) where $P$ is the parity operator, defined by:

$$
(P f)(x)=f(-x), \quad x \in \boldsymbol{R}^{N}, \quad f \in L_{2}\left(\boldsymbol{R}^{N}, d x\right),
$$

more precisely, one has the proposition which is a slight extension of a result of [16]:

Proposition (2.8). Let $\pi$ be a representation of the canonical commutation relations in a Hilbert space $H$, in the sense that

i) the $\pi\left(W_{a}\right)$ are unitary operators

ii) they satisfy the relations (2.3)

iii) $a_{i} \rightarrow \pi\left(W_{a}\right), i=1,2, \cdots, 2 N$ are strongly continuous.

The representation is not necessarily irreducible.

Let $\Phi$ and $\Psi$ be normalized vectors in $H$. Then for any function $f$ on $\boldsymbol{R}^{2 N}$, which is $L_{1}$ as well as its Fourier transform (2.6):

$$
\begin{aligned}
\int_{R^{2 N}} d a & (\Psi f)(a)\left(\Phi \mid \pi\left(W_{a / 2}\right) \Psi\right) \\
& =\int_{R^{2 N}} d a f(a / 2)\left(\Phi \mid \pi\left(W_{a}\right) \tilde{\pi}(P) \Psi\right)
\end{aligned}
$$

where $\tilde{\pi}$ denotes the extension of the representation $\pi$ to the weak closure of the ordinary Schrödinger representation.

Proof goes as follows: If $\pi$ is a Weyl representation of the canonical commutation relations, viz., satisfies i)-iii), then by the Mackey-Von Neumann theorem, there exists a density matrix $\rho$ on the Hilbert space $H_{s}$ of the ordinary Schrödinger representation such that

$$
\begin{gathered}
(\Phi \mid \pi(A) \Phi)=\operatorname{Tr}\{\rho A\}, \\
\text { for all } A \in\left\{\sum_{i=1}^{n} \lambda_{i} W_{a_{i}} ; \lambda_{i} \in C, a_{i} \in R^{2 N}\right\}^{\|\cdot\|}
\end{gathered}
$$

Incidentally that shows that $\pi$ extends to $B\left(H_{s}\right)$, which is the weak closure of $\left\{\sum_{i=1}^{n} \lambda_{i} W_{a_{i}} ; \lambda_{i} \in \boldsymbol{C}, a_{i} \in \boldsymbol{R}^{2 N}\right\}^{\|\cdot\|}$

Let $\mid n) ; n \in Z^{N}$ denotes the orthonormal basis of $H_{s}$ of eigenstates of the harmonic oscillator, then

$$
(\Phi \mid \pi(A) \Phi)=\sum_{m, n \in Z^{N}} \rho_{m, n}(n|A| m)
$$

where 


$$
\rho_{m, n}=(m \mid \rho n) .
$$

But one can compute explicitly that: $(m \geqq n)$

$$
\left(m \mid W_{a} n\right)=\prod_{i=1}^{N} \sqrt{\frac{n_{i} !}{m_{i} !}}\left(\frac{-\alpha_{i}}{\sqrt{2}}\right)^{m_{i}-n_{i}} \exp \left\{\frac{-\left|\alpha_{i}\right|^{2}}{4}\right\} L_{n_{i}}^{m_{2}-n_{i}}\left(\frac{\left|\alpha_{i}\right|^{2}}{4}\right),
$$

where $a=(q, p)$ and

$$
\alpha_{j}=q_{j} \sqrt{M \omega}+i p_{j} \sqrt{\frac{1}{M \omega}},
$$

and $L_{n}^{k}$ denotes the $n$-th Laguerre polynomial of order $k$ (see [17] for definition).

The result follows from an explicit formula about the Fourier transform of Laguerre polynomials (see e.g. [18], p. 42), and the fact that if $P$ is the parity operator

$$
\left.P \mid m)=(-1)^{m} \mid m\right) .
$$

The next observation will be useful: the Weyl operators are the unitary operators which implement translations in phase space, viz.,

$$
W_{a}^{*} Q(f) W_{a}=Q\left(f_{a}\right)
$$

for all $a \in \boldsymbol{R}^{2 N}$ and all functions $f$ on $\boldsymbol{R}^{2 N}$, where

$$
f_{a}(b)=f(b+a) \text {. }
$$

Now let us consider the equilibrium state of the harmonic oscillator at inverse temperature $\beta$

$$
\langle A\rangle_{\beta}=\frac{\operatorname{Tr}\left\{e^{-\beta H_{0}} A\right\}}{\operatorname{Tr}\left\{e^{-\beta H_{0}}\right\}}
$$

for all $A \in B\left(H_{s}\right)$, where $H_{0}$ is given by (2.1).

According to the G. N.S. construction there exists a Hilbert space $H$, a Weyl representation $\pi_{\beta}$ of the canonical commutation relations and a normalized vector $\Omega_{\beta}$ in $H$ which is cyclic for $\tilde{\pi}_{\beta}\left(B\left(H_{s}\right)\right)$ such that

$$
\langle A\rangle_{\beta}=\left(\Omega_{\beta} \mid \tilde{\pi}_{\beta}(A) \Omega_{\beta}\right) \text {, }
$$

where again $\tilde{\pi}_{\beta}$ denotes the extension of the representation $\pi_{\beta}$ to the algebra $B\left(H_{s}\right)$.

However the fundamental relations (2.3)-(2.4) correspond to the value $\hbar=1$ of the Planck's constant. To compute expectation values with the actual value of $\hbar$ one has to compute:

$$
\begin{aligned}
& G(\beta, \hbar, a, f) \\
& \quad=\left(\Omega_{\beta \hbar} \mid \pi_{\beta \hbar}\left(W_{a h^{-1 / 2}}\right) * \pi_{\beta \hbar}\left(Q\left(f^{\hbar^{1 / 2}}\right)\right) \pi_{\beta \hbar}\left(W_{a h^{-1 / 2}}\right) \Omega_{\beta \hbar}\right),
\end{aligned}
$$

where $f^{2}$ denotes the function

$$
f^{\lambda}(a)=f(\lambda a), \quad \lambda>0,
$$

and all $a \in \boldsymbol{R}^{2 N}$.

It is this function which has a path integral representation. Indeed one has:

Proposition (2.21). $G(\beta, \hbar, a, f)$ as a function of $\beta$ and $\hbar$ depends only on $\hbar^{*}$ :

$$
\hbar^{*}=\hbar \operatorname{coth}\left\{\frac{\beta \omega \hbar}{2}\right\},
$$


viz.:

$$
G(\beta, \hbar, a, f)=F\left(\hbar^{*}, a, f\right),
$$

Moreover if $f \in L_{1}\left(\boldsymbol{R}^{2 N}, d x\right)$ :

$$
F\left(\hbar^{*}, a, f\right)=\mathrm{E}\left[f\left(\xi_{a, \hbar^{*}}\right)\right],
$$

where $\xi_{a, \tau}$ is a Wiener process in $\boldsymbol{R}^{2 N}$ starting at a at zero.

Proof relies on the fact that for all $a=(q, p) \in \boldsymbol{R}^{2 N}$,

$$
\left\langle W_{a} P\right\rangle_{\beta}=\left(\operatorname{coth}\left(\frac{\beta \omega}{2}\right)\right)^{-N} \exp \left\{-\frac{1}{4} \operatorname{th}\left(\frac{\beta \omega}{2}\right) \sum_{i=1}^{N}\left\{M \omega q_{i}^{2}+\frac{p_{i}^{2}}{M \omega}\right\}\right\},
$$

on the proposition (2.8) and the well known fact that (2.25) is the transition function of the standard Wiener process.

As a corollary, one can state the following result which is of the type of those obtained for instance in [19], [20].

Corollary (2.26). One has the following limits:

$$
\begin{aligned}
& \lim _{\beta \rightarrow \infty} G(\beta, \hbar, a, f)=\left(0 \mid W_{a \hbar-1 / 2}^{*} Q\left(f^{\hbar^{1 / 2}}\right) W_{a \hbar-1 / 2} 0\right), \\
& \lim _{h \rightarrow 0} G(\beta, \hbar, a, f)=\frac{\int_{R^{2} N} e^{-\beta h_{0}\left(a^{\prime}\right)} f\left(a+a^{\prime}\right) d a^{\prime}}{\int_{R^{2} N} e^{-\beta h_{0}\left(a^{\prime}\right)} d a^{\prime}}, \\
& \lim _{\hbar \rightarrow 0} \lim _{\beta \rightarrow \infty} G(\beta, \hbar, a, f)=f(a),
\end{aligned}
$$

where $h_{0}$ is the classical Hamiltonian function of an harmonic oscillator, viz.:

$$
h_{0}(q, p)=\frac{1}{2} \sum_{i=1}^{N}\left\{\frac{p_{i}^{2}}{M}+M \omega^{2} q_{\imath}^{2}\right\} .
$$

For later purpose it is interesting to consider some linear functionals on $B\left(H_{s}\right)$ which are not positive but which can be used to represent bounded operators on $H_{s}$ as the quantized version of some classical functions on the phase space. Let $A$ be a bounded operator on $H_{s}$; then define:

$$
K(\beta, a, A)=\frac{\operatorname{Tr}\left\{e^{-\beta H_{0}} W_{a}^{*} A W_{a} P\right\}}{\operatorname{Tr}\left\{e^{-\beta H_{0}} P\right\}}
$$

for all $\beta>0$ and $a \in \mathbb{R}^{2 N}$. One has the obvious estimate

$$
|K(\beta, a, A)| \leqq\left\{\operatorname{coth}\left\{\frac{\beta \omega}{2}\right\}\right\}^{N}\|A\|,
$$

and the mapping $a \in \mathbb{R}^{2 N} \rightarrow K(\beta, a, A)$ is obviously continuous.

More precisely one has the following representation for $K(\beta, a, A)$ when $A$ is of the form $Q(f)$ for some $f$.

Proposition (2.33). $K(\beta, a, Q(f))$ has the following path integral representation

$$
K(\beta, a, f)=\mathrm{E}\left[f\left(\xi_{a, \operatorname{th}}\left(\frac{\beta \omega}{2}\right)\right)\right],
$$

consequently 


$$
\lim _{\beta \rightarrow 0} K(\beta, a, f)=f(a) .
$$

This proposition is of practical use, see e.g. [21]. Indeed one has the formula (2.36)

$$
f(a)=\lim _{\beta \rightarrow 0} 2^{N} \operatorname{Tr}\left\{e^{-\beta H_{0}} W_{a}^{*} Q(f) W_{a} P\right\}
$$

which is an easy consequence of the previous proposition and the fact that by an explicit computation

$$
\operatorname{Tr}\left\{e^{-\beta H_{0} P}\right\}=\left(2 \operatorname{ch}\left(\frac{\beta \omega}{2}\right)\right)^{-N}
$$

In order to prove proposition (2.33), one can use the previous formula as well as:

$$
\begin{aligned}
& \operatorname{Tr}\left\{e^{\left.-\beta H_{0} W_{a}\right\}}\right. \\
& \quad=\left(2 \operatorname{sh}\left(\frac{\beta \omega}{2}\right)\right)^{-N} \exp \left\{-\frac{1}{4} \operatorname{coth}\left(\frac{\beta \omega}{2}\right)\left(\sum_{i=1}^{N}\left\{q_{\imath}^{2} M \omega+\frac{p_{i}^{2}}{M \omega}\right\}\right\}\right.
\end{aligned}
$$

for all $a=(q, p) \in \mathbb{R}^{2 N}$, and formulae (2.16) and proposition (2.8).

\section{§3. Temperature Behaviour of Thermal States. Perturbations of the Harmonic Oscillator}

In this section, in some way we want to extend the results of the previous section. However we focus our attention to the temperature dependence of thermal expectation values of systems with $N$ degrees of freedom.

We consider Hamiltonians of the form:

$$
H=\lambda \mathbb{1}+\sum_{\imath=1}^{N}\left\{\frac{1}{2 M} P_{\imath}^{2}+\frac{M \omega^{2}}{2} Q_{\imath}^{2}\right\}+V,
$$

where the potential $V$ is a self-adjoint operator of the form:

$$
V=\int_{R^{2 N}} d \mu(a) W_{a},
$$

$d \mu$ being a bounded measure on $\mathbb{R}^{2 N}$.

In what follows we make use of the following observation: one can change $\lambda$ and $\mu$ in such a way that

$$
\lambda 1+V=\lambda^{\prime} 1+\int_{R^{2 N}} d \mu^{\prime}(a) W_{a}
$$

where $\mu^{\prime}$ is a bounded measure on $R^{2 N}$ and moreover

$$
\int_{R^{2 N}} d \mu^{\prime}(a) W_{a} \geqq 0
$$

Indeed $V$ is a bounded self-adjoint operator, hence by adding a constant we can make it positive. This constant can be incorporated to the measure $\mu$ just adding a mass at zero in the phase space. In the sequel we shall always assume that $V$ is positive.

First we are interested in the matrix elements of the semi-group $\beta \rightarrow \exp \{-\beta H\}$ and our purpose is to derive for these matrix elements a path integral represen- 
tation. The crucial observation is the following lemma:

Lemma (3.5). Let $\pi$ be a Weyl representation of the canonical commutation relations in a Hilbert space $H$. Let $\Phi$ and $\Psi$ be two vectors in $H$ such that

$$
a \in \boldsymbol{R}^{2 N} \longrightarrow F(\beta, a, \Phi, \Psi)=\left(\Phi \mid \tilde{\pi}\left(\exp \{-\beta H\} W_{a}\right) \Psi\right)
$$

is $C_{2}$, where $\tilde{\pi}$ denotes the extension of $\pi$ to $B\left(H_{s}\right)$. Then $F$ satisfies the following integro-differential equation

$$
\begin{aligned}
& \frac{\partial F}{\partial \sigma}(\beta-\sigma, a, \Phi, \Psi) \\
& +\left\{\frac{1}{2 M} \sum_{i=1}^{N} \frac{\partial^{2}}{\partial q_{2}^{2}}+\frac{M \omega^{2}}{2} \sum_{i=1}^{N} \frac{\partial^{2}}{\partial p_{i}^{2}}-\frac{1}{8}\left(\sum_{i=1}^{N} \frac{1}{M} p_{i}^{2}+\sum_{i=1}^{N} M \omega^{2} q_{i}^{2}\right)\right. \\
& \left.\quad-\frac{i}{2}\left(\frac{1}{M} \sum_{i=1}^{N} p_{i} \frac{\partial}{\partial q_{i}}-M \omega^{2} \sum_{i=1}^{N} q_{i} \frac{\partial}{\partial p_{i}}\right)\right\} F(\beta-\sigma, a, \Phi, \Psi) \\
& -\int_{R^{2 N}} d \mu\left(a^{\prime}\right) \exp \left\{-2 i \pi \sigma\left(a, a^{\prime}\right)\right\} F\left(\beta-\sigma, a+a^{\prime}, \Phi, \Psi\right)=0, \\
& a=(q, p) \in \boldsymbol{R}^{2 N}, \quad 0 \leqq \sigma \leqq \beta,
\end{aligned}
$$

with the initial condition:

$$
\lim _{\sigma \rightarrow \beta} F(\beta-\sigma, ; a, \Phi, \Psi)=\left(\Phi \mid \pi\left(W_{a}\right) \Psi\right) .
$$

Proof is a matter of computation.

Using this result one can state the following theorem:

Theorem (3.9). Under the same assumptions as previously and also that if

$$
d \mu(a)=\exp \{i \varphi(a)\} d|\mu|(a)
$$

is the polar decomposition of $\mu$,

i) $|\mu|$ has finite moments up to the second order

ii) $\varphi$ is square integrable with respect to $|\mu|$,

then

$$
\begin{aligned}
& F(\beta, a, \Phi, \Psi) \\
& \quad=\exp \left\{\beta|\mu|\left(\boldsymbol{R}^{2 N}\right)\right\} \mathrm{E}\left[\exp \left\{i \pi \sigma\left(\alpha, \eta_{0}(\beta)\right)\right\} f_{\Phi, \Psi}\left(\xi_{a, 0}(\beta)\right)\right],
\end{aligned}
$$

for all $\beta>0$, and $\alpha \in \boldsymbol{R}^{2 N}$ such that $\alpha_{i}=1, i=1,2, \cdots, 2 N$ 。

$$
f_{\Phi, \Psi}(a)=\left(\Phi \mid \pi\left(W_{a}\right) \Psi\right),
$$

and $\eta_{\tau}, \xi_{a \tau}, \tau>0$ are Markov stochastic processes with values in $R^{2 N}$, which satisfy the following stochastic differential equations:

$$
\begin{aligned}
& \xi_{a, \tau}(\theta)=a+\int_{\tau}^{\theta} d \theta^{\prime} A\left(\xi_{a, \tau}\left(\theta^{\prime}\right), \theta^{\prime}\right) \\
&+\int_{\tau}^{\theta} B\left(\xi_{a, \tau}\left(\theta^{\prime}\right), \theta^{\prime}\right) d W_{\theta^{\prime}}+\int_{\tau}^{\theta} \int_{R^{2 N}} C\left(\xi_{a, \tau}\left(\theta^{\prime}\right), \theta^{\prime}, u\right) \tilde{\nu}\left(d \theta^{\prime}, d u\right), \\
& \eta_{\tau}(\theta)=\int_{\tau}^{\theta} d \theta^{\prime} A^{\prime}\left(\xi_{a, \tau}\left(\theta^{\prime}\right), \theta^{\prime}\right)
\end{aligned}
$$




$$
+\int_{\tau}^{\theta} B^{\prime}\left(\xi_{a, \tau}\left(\theta^{\prime}\right), \theta^{\prime}\right) d W_{\theta^{\prime}}+\int_{\tau}^{\theta} \int_{R^{2 N}} C^{\prime}\left(\xi_{a, \tau}\left(\theta^{\prime}\right), \theta^{\prime}, u\right) \tilde{\mathcal{\nu}}\left(d \theta^{\prime}, d u\right),
$$

where the $W \equiv\left(W_{i}\right)_{i=1,2, \cdots, 2 N}$ are $2 N$ independent Wiener processes, $\tilde{\nu}(d \theta, d u)$ is a standard Poisson measure on $\boldsymbol{R}^{2 N}$ such that

$$
\tilde{\nu}(\theta, D)=\nu(\theta, D)-\theta i \mu \mid(D),
$$

and

(3.16) $\quad \mathrm{E}[\nu(\theta, D)]=\theta|\mu|(D) \quad \theta>0$ and $D$ a Borel set in $\boldsymbol{R}^{2 N}$.

Furthermore if $a=(q, p) \in \boldsymbol{R}^{2 N}$

$$
\begin{aligned}
& A(a, t)_{i}=\int_{R^{2 N}} a_{i}^{\prime} d|\mu|\left(a^{\prime}\right) \quad i=1,2, \cdots, 2 N \\
& A^{\prime}(a, t)_{i}=\int_{R^{2 N}}\left\{\frac{\varphi\left(a^{\prime}\right)}{N}+q_{i} p_{i}^{\prime}+\frac{\pi}{N}\right\} d|\mu|\left(a^{\prime}\right) \quad i=1,2, \cdots, N \\
& A^{\prime}(a, t)_{N+i}=\int_{R^{2 N}}\left\{-\frac{\varphi\left(a^{\prime}\right)}{N}+q_{i}^{\prime} p_{i}-\frac{\pi}{N}\right\} d|\mu|\left(a^{\prime}\right) \quad i=1,2, \cdots, N \\
& B(a, t)_{i, j}=\frac{1}{\sqrt{M}} \delta_{i, j}, \quad i=1,2, \cdots, N, \quad j=1,2, \cdots, 2 N, \\
& B(a, t)_{i j}=\omega \sqrt{M} \delta_{i, j}, \quad i=N+1, \cdots, 2 N, \quad j=1,2, \cdots, 2 N \\
& B^{\prime}(a, t)_{i j}=-\frac{1}{\sqrt{M}} p_{i} \delta_{i, j}, \quad i=1,2, \cdots, N, \quad j=1,2, \cdots, 2 N \text {, } \\
& B^{\prime}(a, t)_{i j}=-\omega \sqrt{M} \delta_{i, j}, \quad i=N+1, \cdots, 2 N, \quad j=1,2, \cdots, 2 N, \\
& C\left(a, t, a^{\prime}\right)=a^{\prime} \text {, } \\
& C^{\prime}\left(a, t, a^{\prime}\right)_{i}=+\frac{\varphi\left(a^{\prime}\right)}{N}+p_{i}^{\prime} q_{i}+\frac{\pi}{N}, \quad i=1,2, \cdots, N, \\
& C^{\prime}\left(a, t, a^{\prime}\right)_{N+i}=-\frac{\varphi\left(a^{\prime}\right)}{N}+p_{i} q_{i}^{\prime}-\frac{\pi}{N}, \quad i=1,2, \cdots, N \text {, }
\end{aligned}
$$

Proof. Previous statement is based on the observation that (3.7) is the backward Kolmogorov equation for the system of stochastic differential equations (3.13) and (3.14), (see [22], chap. 2, §10). Then it is rather easy but tedious to check that formulae (3.17)-(3.22) give the equation (3.7) as the backward Kolmogorov equation associated with the stochastic differential equations (3.13) and (3.14) (see [22], p. 302).

The previous theorem cannot be extended directly to the thermal functionals

$$
a \in \boldsymbol{R}^{2 N} \longrightarrow \operatorname{Tr}\left\{e^{-\beta H} W_{a}^{*} A\right\},
$$

for those $A$ which are not of trace class. However one can expect on the basis of the results of Section 2 that this can be done for those of the type:

$$
a \in \boldsymbol{R}^{2 N} \longrightarrow \operatorname{Tr}\left\{e^{-\beta H} W_{a}^{*} Q(f) P\right\} .
$$

For high temperature one can expect that the effect of the interaction is small. This is the case as we can see by a series of easy results. 
Lemma (3.25). Let $H$ and $H_{0}$ be as in (3.1), then

$$
\lim _{\beta \rightarrow 0} \frac{\operatorname{Tr}\left\{e^{-\beta H}\right\}}{\operatorname{Tr}\left\{e^{-\beta H_{0}}\right\}}=1 \text {, }
$$

Proof. Applying twice the inequality (see e.g. [23])

$$
\operatorname{Tr}\{A-B\} \leqq \operatorname{Tr}\{A \operatorname{Ln}(A)-A \operatorname{Ln}(B)\},
$$

putting $\quad A=\exp \left(-\beta H_{0}-\beta \lambda\right), \quad B=\exp (-\beta H), \quad($ resp. $\quad A=\exp (-\beta H), \quad B=$ $\left.\exp \left(-\beta H_{0}-\beta \lambda\right)\right)$ gives

$$
\beta \frac{\operatorname{Tr}\left\{e^{-\beta H} V\right\}}{\operatorname{Tr}\left\{e^{-\beta H_{0}-\beta \lambda}\right\}} \leqq 1-\frac{\operatorname{Tr}\left\{e^{-\beta H}\right\}}{\operatorname{Tr}\left\{e^{-\beta H_{0}-\beta \lambda}\right\}} \leqq \beta \frac{\operatorname{Tr}\left\{e^{-\beta H_{0}} V\right\}}{\operatorname{Tr}\left\{e^{-\beta H_{0}}\right\}} .
$$

However we have assumed (by a suitable choice of $\lambda$ ) that $V$ is positive, hence the left hand side is positive. Furthermore the right hand side is obviously bounded by $\beta\|V\|$. This concludes the proof.

Lemma (3.28). With the same assumptions as above

$$
\left|\frac{\operatorname{Tr}\left\{e^{-\beta H} A\right\}}{\operatorname{Tr}\left\{e^{-\beta H}\right\}}-\frac{\operatorname{Tr}\left\{e^{-\beta H_{0}} A\right\}}{\operatorname{Tr}\left\{e^{-\beta H_{0}}\right\}}\right| \leqq 2 \beta\|A\| \cdot\|V\|,
$$

for all $\beta>0, A \in B\left(H_{s}\right)$.

Proof. One has the obvious inequality

$$
\begin{aligned}
& \left|\frac{\operatorname{Tr}\left\{e^{-\beta H} A\right\}}{\operatorname{Tr}\left\{e^{-\beta H}\right\}}-\frac{\operatorname{Tr}\left\{e^{-\beta H_{0}} A\right\}}{\operatorname{Tr}\left\{e^{-\beta H_{0}}\right\}}\right| \\
& \leqq \\
& \quad\left|\frac{\operatorname{Tr}\left\{e^{-\beta H} A\right\}}{\operatorname{Tr}\left\{e^{-\beta H}\right\}}\right|\left|\left(1-\frac{\operatorname{Tr}\left\{e^{-\beta H}\right\}}{\operatorname{Tr}\left\{e^{-\beta H_{0}-\beta \lambda}\right\}}\right)\right| \\
& \quad+\left|\frac{\operatorname{Tr}\left\{\left(e^{-\beta H}-e^{-\beta H_{0}-\beta \lambda}\right) A\right\}}{\operatorname{Tr}\left\{e^{-\beta H_{0}-\beta \lambda}\right\}}\right| .
\end{aligned}
$$

The first term in the right hand side is bounded by $\beta\|V\| \cdot\|A\|$. The second term can be rewritten

$$
\begin{aligned}
& \left|\frac{\operatorname{Tr}\left\{\left(e^{-\beta H}-e^{-\beta\left(H_{0}+\lambda\right)}\right) A\right\}}{\operatorname{Tr}\left\{e^{-\beta\left(H_{0}+\lambda\right)}\right\}}\right| \\
& \quad \leqq\left|\int_{0}^{\beta} d \gamma \frac{\operatorname{Tr}\left\{e^{-\beta\left(H_{0}+\lambda\right)} e^{\gamma\left(H_{0}+\lambda\right)} V e^{-\gamma H} A\right\}}{\operatorname{Tr}\left\{e^{-\beta\left(H_{0}+\lambda\right)}\right\}}\right| .
\end{aligned}
$$

A standard argument shows that it is bounded by $\beta\|A\| \cdot\|V\|$. This concludes the proof.

However we proved in Section 2 that:

$$
\lim _{\beta \rightarrow 0} 2^{N} \operatorname{Tr}\left\{e^{-\beta H_{0}} W_{a}^{*} Q(f) W_{a} P\right\}=f(a) .
$$

Consequently we can state the proposition:

Proposition (3.29). Let $f$ be a twice differentiable $L_{1}$ function on the phase space. Then one has the following path integral representation: 


$$
\begin{aligned}
& \operatorname{Tr}\left\{e^{-\beta H} W_{a}^{*} Q(f) P\right\} \\
& \quad=2^{-N} \exp \left\{\beta|\mu|\left(\mathbb{R}^{2 N}\right)\right\} \mathrm{E}\left[\exp \left(i \pi \sigma\left(\alpha, \eta_{0}(\beta)\right) f\left(\xi_{a, 0}(\beta)\right)\right]\right.
\end{aligned}
$$

where $\xi_{a-}, \eta_{\tau}$ are the Markov processes defined in Theorem (3.9).

Proof is an easy consequence of the previous result if one observes moreover that from (2.36)

$$
\lim _{\beta \rightarrow 0} 2^{N} \operatorname{Tr}\left\{e^{\beta\left(H_{0}+\lambda\right)} Q(f) P\right\}=f(0)
$$

and that from (2.3) and (2.5)

$$
W_{a}^{*} Q(f)=Q\left(g_{a}\right), \quad a \in \boldsymbol{R}^{2 N}
$$

where

$$
g_{a}(b)=f(a+b) \exp (-4 i \pi \sigma(a, b))
$$

for all $b \in \mathbb{R}^{2 N}$.

\section{References}

[1] Ginibre, J., Mécanique Statistique et Théorie des Champs, Les Houches (1970), C. de Witt, R. Stora Eds. Gordon and Breach, (1971), 327-427.

[2] Hфegh-Krohn, R., Commun. Math. Phys. 38 (1974), 195-224.

[3] Fröhlich, J., Helv. Phys. Acta 48 (1975), 355-368.

[4] Klein, A., New Stochastic Methods in Physics, Les Houches (1980), C. de WittMorette, K.D. Elworthy Eds., Physics Reports 77, (1981), 329-337.

[5] Donsker, M.D. and Varadhan, S.R.S., The Polaron Model, to appear. See also ref. [4], p. 235-237.

[6 ] De Witt-Morette, C., Maheshwari, A. and Nelson, B., Phys. Reports, 50 (1979), 255-372.

[7] Glimm, J. and Jaffe, A., Quantum Physics, Springer Verlag, 1981.

[8] Feynman, R.P., Rev. Mod. Phys. 20 (1948), 367-387.

[9] Feynman, R.P. and Hibbs, A.R., Quantum Mechanics and Path Integrals, McGraw-Hill, 1965.

[10] Maslov, V.P. and Chebotarev, A.M., Itogi Nauki i Tekhniki 15 (1978), 5-78.

[11] Combe, Ph., Hфegh-Krohn, R., Rodriguez, R., Sirugue, M. and Sirugue-Collin, M.. Commun. Math. Phys., 77 (1980), 269-288.

[12] — - - $\longrightarrow$, - Feynman Path Integrals with Piecewise Classical Paths, J. Math. Phys. 23 (1982), 405.

[13] Fano, U., Rev. Mod. Phys., 29 (1957), 74-93.

[14] Albeverio, S., Blanchard, Ph., Combe, Ph. and Sirugue, M., Relativistic Invariant Flows for Quantum Fields, Preprint Marseille, CPT-81/P. 1342 (1981).

[15] Daubechies, I., J. Math. Phys., 21 (1980), 1377-1389.

[16] Grossmann, A., Commun. Math. Phys. 48 (1976), 191-194.

[17] Bateman, H., Higher Transcendental Functions, 2, McGraw-Hill, 1953.

[18] - Tables of Integral Transform, 2, MicGraw-Hill, 1954.

[19] Hepp, K., Commun. Math. Phys., 35 (1974), 265-277.

[20] Grossmann, A. and Seiler, R., Commun. Math. Phys. 48, (1976), 195-197.

[21] Combe, Ph., Rodriguez, R., Sirugue, M. and Sirugue-Collin, M., Group Theoretical Methods in Physics, Montréal (1976), R. Sharp, B. Kolman Eds. Academic Press, 1977, 349-359.

[22] Gihman, I.I. and Skorohod, A.V., Stochastic Differential Equations, SpringerVerlag, 1972.

[23] Ruelle, D., Statistical Mechanics, W. Benjamin, 1969. 
